5. The following are commonly associated:

a sedation and treatment with chlorpromazine

b neuroleptic malignant syndrome and treatment with chlorpromazine

c antipsychotic treatment and respiratory depression

d droperidol treatment and akathisia

e IM antipsychotic treatment and sudden unexplained death

\begin{tabular}{|c|c|c|c|c|}
\hline \multicolumn{5}{|c|}{ MCQ answers } \\
\hline 1 & 2 & 3 & 4 & 5 \\
\hline a $T$ & a $\mathrm{T}$ & a $T$ & a $T$ & a $\mathrm{T}$ \\
\hline b F & b F & b F & b $T$ & b F \\
\hline c $T$ & c $F$ & c $T$ & c $\mathrm{T}$ & c $F$ \\
\hline d F & d F & d T & d $T$ & d $T$ \\
\hline e $T$ & e $F$ & e $F$ & e $F$ & e $F$ \\
\hline
\end{tabular}

\title{
Comment
}

\section{Trevor Turner}

There has long been considerable individual variation in the regimes for managing acutely disturbed patients, in terms of the drugs used, routes of administration and the dosages given. We all tend to learn from 'accepted' practice in the units in which we train, and the lack of good-quality controlled trials, published in leading and accessible journals, compounds the problem. Given the importance of this issue, when concerns about sudden death in the context of high dose regimes are generating considerable publicity (for example, MIND is calling for a blanket ban on dosages greater than those given in the BNF), the need for rigorous research has never been greater. Guidelines have, however, become established in a number of units, and this clear summary by Macpherson $e t$ al is therefore to be welcomed.

The authors have, understandably, concentrated on medication issues but their comments on staffing and environment are equally pertinent. There is evidence that increased proportions of agency staff are associated with increased levels of violence on a unit. Training and experience, as well as personal knowledge of patients (and vice versa) when they are well, can often prevent outbursts or alleviate disturbed situations. Being aware of predictors of violence in terms of previous history, symptoms, and levels of arousal (e.g. posture, sweating/respiration, eye-contact) can ensure appropriate staffing levels and the early use of medication. We probably also undervalue the impact of environment. In Hackney our untoward incident rates on the wards, and the use of Section 5 (2), dropped by up to $50 \%$ when we moved from an old workhouse unit to new, purpose-built wards built around the principles of 'space' and 'light'.

In terms of the practical aspects of what to do, it may often seem impossible to carry out a true mental state assessment on a mute or screaming patient who is being restrained in a side room. Inexperienced trainees may feel that trying to clarify symptoms, while frustrated nurses are hanging on to the patient and expecting something to be done, is not worth the delay. Such assessments need a fine judgement, and an immediate clarification of who is in charge of a disturbed incident. Explaining to all, amidst a mêleee, what you are doing, why you are doing it, and what the treatment plan will be, can ensure good team work and coordinated activity. Visitors, other patients and family usually need to be kept out of the way. The straightforward act of constantly reassuring the patient that you are doctors and nurses and here to help, seems obvious but is often forgotten.

Trevor Turner was appointed Consultant Psychiatrist at St Bartholomews' and Hackney Hospitals in 1987. He has a special interest in the problems of acute care in the 'inner city', with publications on Section 136, Community Care and the management of schizophrenia. He is currently Medical Director of the City and Hackney Community Services NHS Trust. 
Documenting behavioural and mental state details, treatment plans and medications given in clear timed and dated notes is essential. It also gives one time to think, to clarify treatment steps, for example while awaiting responses to medication, and to ensure that other colleagues can easily acquaint themselves with what has happened. The doctor supervising acutely disturbed patients should stay on hand until the nurses feel assured that the situation is safe.

Given that most disturbed patients are well known to treatment units, and will not be neuroleptic naive, it is vital that notes or summaries are easily accessible. We regularly reinstate depot, along with Acuphase and lorazepam if necessary, when a potentially violent patient is admitted on relapse. Two or three days relative sedation, under good observation, is in the best interests of the patient, the family and the staff. The plea of an old patient, "doctor, don't let me get like that again", indicates the mental anguish of manic or confused sufferers. Likewise the use of liquid oral preparations, rather than tablets, for the suspiciously hostile, or the use of Acuphase (we have used it even at home with the support of the GP, community nurses and the willing family) can ensure continued stabilisation.

Crucially, these guidelines outline the key need for much better audit and research of acute treatment approaches, and should be most useful in generating this process. Whether, however, we can provide the necessary numbers and quality of trained staff, in properly designed and therapeutic environments, is unfortunately much less certain. Resource limitations abound, and the shortage of trained mental health nurses requires thoughtful recruitment policies locally and nationally. Additional pay scales for staff in psychiatric intensive care units will have to equal the 'forensic' lead available in most medium secure units now. Historically psychiatry has been judged by its management of the 'furiously mad', and intensive care unit experience should be a sine qua non of both medical and nursing training. 